\title{
Individualised Funding: A Realist Analysis to Identify the Causal Factors That Facilitate Positive Outcomes
}

\author{
Padraic Fleming ${ }^{1,2, * \mathbb{D}}$, Sinead McGilloway ${ }^{2}$ and Steve Thomas $^{1}(\mathbb{D}$ \\ 1 Centre for Health Policy and Management, Trinity College Dublin, D02 PN40 Dublin, Ireland; \\ thomassd@tcd.ie \\ 2 Centre for Mental Health and Community Research, Department of Psychology and Social Sciences Institute, \\ Maynooth University, W23 F2H6 Maynooth, Ireland; sinead.mcgilloway@mu.ie \\ * Correspondence: padraic.fleming@tcd.ie
}

check for updates

Citation: Fleming, P.; McGilloway, S.; Thomas, S. Individualised Funding: A Realist Analysis to Identify the Causal Factors That Facilitate Positive Outcomes. Disabilities 2021, 1, 47-57. https://doi.org/10.3390/ disabilities1010004

Received: 25 January 2021

Accepted: 23 February 2021

Published: 26 February 2021

Publisher's Note: MDPI stays neutral with regard to jurisdictional claims in published maps and institutional affiliations.

Copyright: (c) 2021 by the authors. Licensee MDPI, Basel, Switzerland. This article is an open access article distributed under the terms and conditions of the Creative Commons Attribution (CC BY) license (https:// creativecommons.org/licenses/by/ $4.0 /)$.

\begin{abstract}
There is growing interest, internationally, in empowering people with disabilities, and the United Nations have identified individualised funding as one way in which empowerment might be achieved. 'Individualised funding' is an umbrella term for various publicly funded support structures that provide personalised and individualised support services for people with a disability. These aim to facilitate self-direction, empowerment, independence, and self-determination. The findings of a recent mixed-methods systematic review of studies undertaken during an approximate 25-year period suggest positive effects with respect to quality of life, client satisfaction, and safety, as well as very few adverse effects, although the evidence on cost-effectiveness was inconclusive. This paper involved a re-examination of the qualitative findings of that review by employing a realist framework to explore the interplay between key contexts and mechanisms, and how these facilitate or inhibit positive outcomes associated with individualised funding and its underlying programme theory.
\end{abstract}

Keywords: individualised funding; personal budgets; self-determination; empowerment; disability; realist; CMOC; implementation

\section{Introduction}

The United Nations Convention on the Rights of Persons with a Disability recognises 'personal budgets' as a means to achieving self-determination for people with a disability, who, according to World Health Organisation estimates, account for almost $15 \%$ of the world's population [1,2]. The Convention reinforced a growing policy shift internationally, towards people with a disability living autonomous, self-determined lives whereby they are empowered and can live as independently as possible, choosing their own supports and self-directing their lives $[3,4]$. Personal budgets are just one example of the many funding and support structures that are captured under the umbrella term of individualised funding' — an approach designed to provide personalised and self-directed supports for people with a disability, which places individuals at the centre of decision-making around how and when they are supported [5].

Individualised funding can take many forms including, in particular, (a) directpayments, whereby funds are given directly to the person with a disability who then self-manages this money to meet their individual needs; (b) a microboard, brokerage model, or 'managed' personal budget that provides a similar amount of freedom for the person with a disability, but an intermediary service offers administrative support, while providing guidance and information; and (c) the Cash and Counselling model, predominantly found in the US, represents a combination of the direct payment and intermediary models, allowing the user the flexibility to choose between a self-managed and a professionally managed support service [5-7]. The type of model available is often dictated by policy makers, but other considerations include the type of disability or available support 
networks. Direct payments, for example, often involve considerable administrative duties and are more likely, therefore, to be utilised by people with a physical or sensory disability and less so by those with an intellectual or developmental disability-although this is not universally true.

The common programme theory underpinning all of these models (i.e., what individualised funding is expected to achieve) is to facilitate self-direction, empowerment, independence, and self-determination for people with a disability. Despite these aspirations, early studies in the US, the UK, and Europe highlighted potential risks associated with the marketisation and indirect privatisation of care services, whereby people with a disability or 'consumers of care' increasingly act as employers without necessarily having the human resource skills or knowledge of available care choices $[8,9]$. Conversely, more recent evidence suggests that people availing of individualised funding are capable of acquiring the necessary skills, or indeed are able to outsource certain tasks in order to successfully bypass the service providers and contract their support services directly [10].

We conducted a recent mixed methods systematic review to contribute to the ongoing debate in this field and to systematically capture and assess the growing pool of evidence related to individualised funding (for people aged 18 and over with a lifelong disability). The aims of this review, which was conducted in two phases, were to: (1) determine the effectiveness of individualised funding in relation to health and social care outcomes; and (2) to critically appraise and synthesise the qualitative evidence relating to stakeholder perspectives and experiences of individualised funding. The review sought to focus on 'initial implementation' as described by Fixsen, Naoom, Blase, Friedman, and Wallace, as it is often the most challenging stage of implementation, requiring ongoing and multi-level change (e.g., individual, environmental, and organisational), before the next stage (full operation) can be successfully initiated [11]. This work was conducted in accordance with Campbell Collaboration guidelines [12].

The first (effectiveness) question was addressed by reviewing seven unique studies (linked to a total of 19 published and unpublished titles) that compared people in receipt of individualised funding with a control group. As such, single-case designs, pre-post studies without a control group, non-matched control groups, or groups matched in a post hoc way after results were known were excluded from the review. The control group could consist of: (a) traditional 'services as usual' (e.g., predetermined group activities provided in a congregated setting and financed through block funding to service providers); or (b) a different type of personalised support which does not include a personal budget. A total of 35 measures were used within these studies to assess the various health and social care outcomes of interest, such as quality of life and client satisfaction. Most of the outcomes of interest were reported in two studies (four and five, respectively) [13,14]. The remaining five studies incorporated between one and three outcomes of interest. For the studies that found statistically significant between-group differences across the relevant health and social care outcomes reported, most were in favour of the intervention group. The most consistently positive outcomes related to quality of life and client satisfaction. There was also evidence to suggest fewer adverse effects for those in receipt of individualised funding. There was less evidence of impact for physical functioning, unmet need and cost effectiveness [15].

The second phase — the qualitative synthesis of 69 studies [15] — was informed by, and conducted within, a realist evaluation framework which considers 'Contexts, Mechanisms and Outcomes' (CMOs) [16]. This paper seeks to re-examine this work by refining the initial programme theory of individualised funding, using the contexts and mechanisms identified in the review to construct a generative model of causality (CMO configurations); this, in turn, was used to identify how, why, and to what extent individualised funding improves self-direction, empowerment, independence, and self-determination [16]. The overall aim is to better understand the complex interplay between the contexts and mechanisms that lead to the successful implementation of individualised funding and which, therefore, impact on its overall effectiveness. 
According to Jagosh [17], 'context' can be interpreted as anything in the backdrop that may not formally be part of, but can impact upon, the intervention, such as cultural norms/values, history, and existing public policy or economic conditions. 'Mechanisms' may be defined as more specific underlying entities, processes, or structures that lead to particular outcomes [18]. For example, in the case of complex social interventions, mechanisms can be a cognitive process which stimulates or demotivates stakeholders, including those delivering the intervention [17]. Context and mechanisms can, therefore, affect the outcomes or effectiveness of an intervention.

As Wong and Westhorp et al. [19] explained, a CMO configuration explores the relationship between particular features of context (C) and mechanisms (M) (in this case during the implementation of individualised funding) that lead to certain outcomes $(\mathrm{O})$. These outcomes may relate to the health and social care benefits reported, or they may indeed point to new (meso or micro) outcomes that contribute to the overall programme success - this can be intended or unexpected. CMO configurations may be represented by a statement, diagram or drawing. They may pertain to individualised funding (the programme) as a whole, or to specific aspects of the intervention. One CMO may be embedded in another or configured in a series, along the implementation process, from early exploration, through initial implementation, and finally full implementation, whereby they can inform the long-term sustainability of individualised funding.

Thus, the examination of $\mathrm{CMO}$ configurations can help to develop an understanding of how a particular context acts on a specific program mechanism to produce outcomes and how, therefore, it can modify the effectiveness of an intervention.

\section{Materials and Methods}

This section summarises the methods related to the qualitative element of the systematic review, within which this current study is framed. The data pertained to a 24 -year period from 1992 to 2016 . The qualitative meta-synthesis presented the experiences of individuals participating in an individualised funding intervention, as well as documenting implementation successes and challenges from the perspective of multiple stakeholders.

\subsection{Search Strategy}

Ten academic databases and nine other grey literature databases/search engines were utilised (CINAHL, EMBASE, Medline First Search, ASSIA, PsycInfo, SCOPUS, Sociological Abstracts, Worldwide Political Science Abstracts, EconLit, Business Source Complete, Greylit, OpenGrey, ProQuest Dissertations and Theses, Google Scholar, Google, Australian Policy Online, VHL Regional Portal, NORART, and Theses Canada). The terms used to customise the search string for specific databases were based on the 'population' and 'intervention' of interest. 'Disability' and all possible variations, including mental health, disorders, and autism, was the first keyword. 'Budget' and all variations thereof was the second keyword. Database-specific conventions were followed to 'explode' or 'truncate' key terms as appropriate. A list of free-text terms identified from the literature were used to supplement the syntax developed. Study design and outcomes were not included as part of the search strategy, as it was anticipated that this would potentially lead to the omission of relevant literature. Bibliographies from included and some excluded studies (e.g., literature reviews) were used to guide forward citation searching. Conference proceedings, manual browsing of key journals, and other online materials were used to guide hand-searching.

\subsection{Selection Criteria}

The population of interest included: adults (aged 18 years and over) receiving a personal budget, with any form or level of lifelong disability (physical, sensory, intellectual, or developmental disability, level of mental health problem, disorder or illness, or dementia), residing in any country and any type of residential setting (own home, group home, residential care setting, nursing home, hospital, institution). Studies in any language were included. 
Minors and older people without a lifelong disability (i.e., no disability in 10 years prior to reaching the age of 65) were excluded, as were privately funded individualised funding interventions. Stakeholders included the client, family members, advocates, personal assistants/key workers, professional staff such as occupational therapists or physiotherapists, and other members of the community involved in the process.

\subsection{Data Screening}

The screening of studies was undertaken independently by three researchers and involved, firstly, title and abstract screening $(n=7158)$ and, secondly, full text documents $(n=328)$. After full text screening, 215 studies were excluded-33\% due to the nature of the intervention, $20 \%$ based on study design, $19 \%$ due to a lack of empirical data, $13 \%$ because they did not include the population of interest, $13 \%$ because they did not report the outcomes of interest, and finally four studies for which full texts could not be located $(2 \%)$. A further 40 titles were not unique studies but were reviewed in conjunction with the 73 unique studies included in the review ( 3 of which were mixed methods). Risk of bias and the quality of the research were evaluated by one reviewer using a range of tools (depending on study design) from the Critical Appraisal Skills Programme (CASP); studies were also assessed using the CerQual headings of: 'methodological limitations', 'relevance', 'adequacy of data' and 'coherence'. Further quality checks took place, during full text screening, by two of the reviewers.

\subsection{Synthesis of Qualitative Results}

The meta-synthesis of qualitative data was conducted in two stages and informed by, and conducted within, a realist evaluation framework which considers 'Contexts, Mechanisms and Outcomes' [16] to understand how the implementation of individualised funding can impact the attainment of positive outcomes. During stage one of the thematic analysis (reading and coding the studies), five overarching themes were identified related to implementation including: positive experiences; negative experiences; potential for adverse effects; contributory factors; and process. At the end of stage one, there were 18,279 individually coded pieces of text, representing 696 possible individual micro-themes. Any unexpected or emergent themes were examined to ensure conceptual agreement between reviewers.

During stage two, the themes were refined by exploring relationships between the codes. Once studies had been conceptually folded together, a final set of themes were categorised using Bronfenbrenner's 'macro to micro' framework, an approach adopted previously by Fleming et al. [20] and Laragy and Ottmann [21]. MAXMaps, an analytical tool within the qualitative analysis software MAXQDA [22], was used to examine relationships between codes and, in particular, 'co-occurring codes', which relate to pieces of text that had two or more codes assigned to them.

The remainder of this paper will focus on the most important CMO configurations that emerged from this analysis, based on information power, i.e., the evidence that was considered most useful in terms of the explanatory and theoretical aims of this study, based on strong and insightful empirical dialogue extracted from primary studies [23].

\section{Results}

The views of over 9000 people were captured in the 69 qualitative studies (Table 1), representing a total of 96 published and unpublished titles (Supplementary Materials), $73 \%$ of which were from the perspective of individuals with a disability or their representative. As with the quantitative findings, the intervention was positively received overall, despite, amongst other things, considerable issues in accessing funding, implementation challenges, and process delays. Most people, even those who were somewhat aggrieved, reported that they preferred the intervention over traditional service provision [15]. 
Table 1. Summary of qualitative study characteristics.

\begin{tabular}{lccc}
\hline \multicolumn{1}{c}{ Characteristic } & 69 Studies (\%) & Characteristic & 69 Studies (\%) \\
\hline Publication Year & $6(9)$ & UK & $41(59)$ \\
\hline $1992-1999$ & $16(23)$ & United States/Canada & $17(25)$ \\
\hline $2000-2005$ & $23(33)$ & Australia & $7(10)$ \\
\hline $2006-2010$ & $24(35)$ & Other European & $4(6)$ \\
\hline $2010-2016$ & \multicolumn{3}{c}{ Disability Type } \\
\hline Intervention Type & $21(30)$ & Various & $41(59)$ \\
\hline Direct/In-direct payment & $12(17)$ & Mental Health/Dementia & $10(14)$ \\
\hline $\begin{array}{l}\text { Self-directed/determination/ } \\
\text { managed }\end{array}$ & $12(17)$ & Physical/Sensory & $7(10)$ \\
\hline Personal budget & $7(10)$ & Learning & $5(7)$ \\
\hline Individual budget & $17(25)$ & Not specified & $6(9)$ \\
\hline Mixed/Other & & Sample Size \\
\hline Study Design & $20(29)$ & $<25$ & $24(35)$ \\
\hline In-depth interviews & $19(28)$ & $26-50$ & $16(23)$ \\
\hline Mixed qualitative & $18(26)$ & $51-100$ & $16(23)$ \\
\hline Case study & $9(13)$ & $\geq 101$ & $13(19)$ \\
\hline Survey & $3(4)$ & & \\
\hline Other & $68(99)$ & & \\
\hline Language & $1(1)$ & & \\
\hline English & & \\
\hline Non-English & & \\
\hline
\end{tabular}

The meta-synthesis demonstrated that a wide range of perceived benefits were consistently reported across jurisdictions and various models of individualised funding. While the review also examined successes and challenges from the perspective of staff, this paper primarily focuses on the views of services users and their representatives. Perceived benefits, many of which were inter-related, included freedom, flexibility, continuity of care/service/life, improved family life and social opportunities, and enhanced self-image. Whilst these may be considered positive outcomes associated with access to individualised funding, it may be more informative to consider them as mechanisms within the CMO configurations.

For example, 'freedom' was the most widely cited benefit identified in the metasynthesis, representing $23 \%$ (773) of the coded pieces of text. Study participants highlighted the freedom to choose who supports them, as well as how, when, and where the support is provided. Freedom was often inextricably linked to flexibility that had previously been absent from traditional, often 'group-based' structures of support. Having control around life choices was a facilitative mechanism in itself. Freedom (to choose) also extended to personal freedoms such as 'perceived autonomy', 'self-determination', 'self-direction', 'selfreliance', 'sense of empowerment', 'space and freedom', and 'freedom to make mistakes'. Perhaps in this context, enhanced agency takes the form of a mechanism that leads to another outcome- 'improved self-image'. This interpretation aligns with the notion that mechanisms not only relate to new ways of doing things, as a result of an intervention, but also how participants reason with, and understand, these changes [16] (Figure 1). 


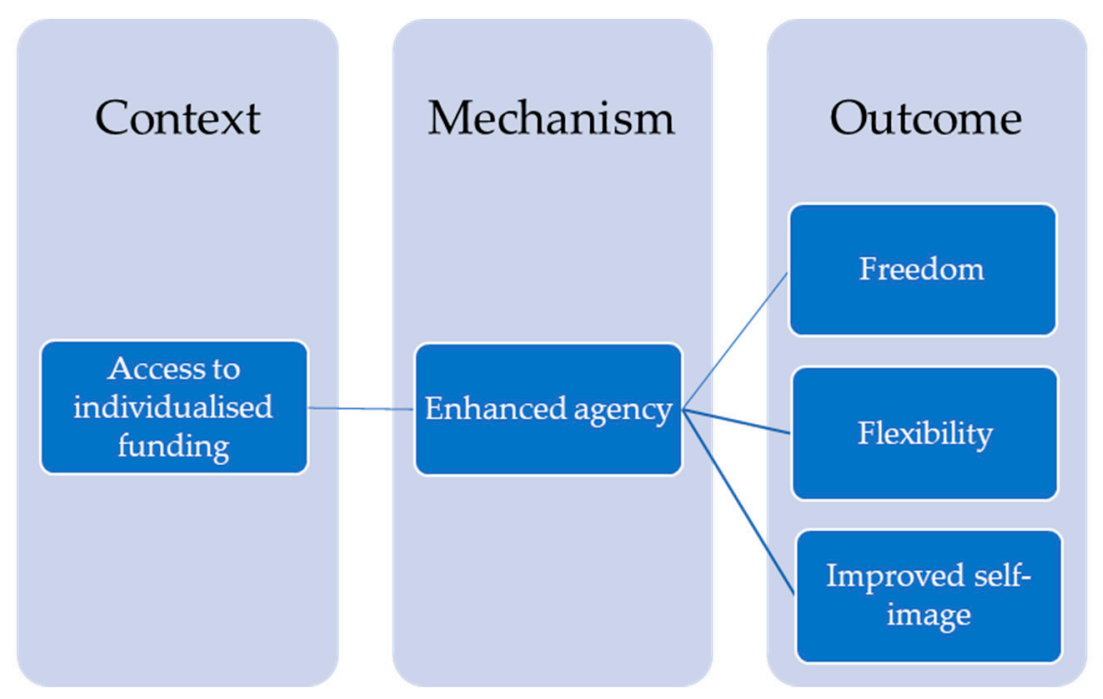

Figure 1. Macro ‘Contexts, Mechanisms and Outcomes' (CMO) configuration.

An important theme that emerged from the literature, and one also linked to greater control, was the 'shift in power' from agencies/organisations to the person with a disability or their representative. In many ways, this was seen as transformative in terms of how people could live their lives-arguably becoming needs-led in the true sense. With this contextual shift, participants reported feeling more resilient with a feeling of greater control over the decisions that impact their day-to-day lives; this ultimately helped to promote their self-image, self-belief, and self-esteem, which, in turn, were associated with other positive outcomes, such as greater confidence, hope, and a more positive outlook in life as well as lower levels of stress and anxiety.

With this shift in power also came a shift in responsibilities for many individuals, particularly those who were self-managing their funding; the evidence indicated that this was often associated with the complex processes involved in accessing and managing an individualised fund. Another, perhaps unexpected, outcome was increased value for money, with people reporting the freedom to shop around for the best value, or indeed generating savings by removing an intermediary (Figure 2).

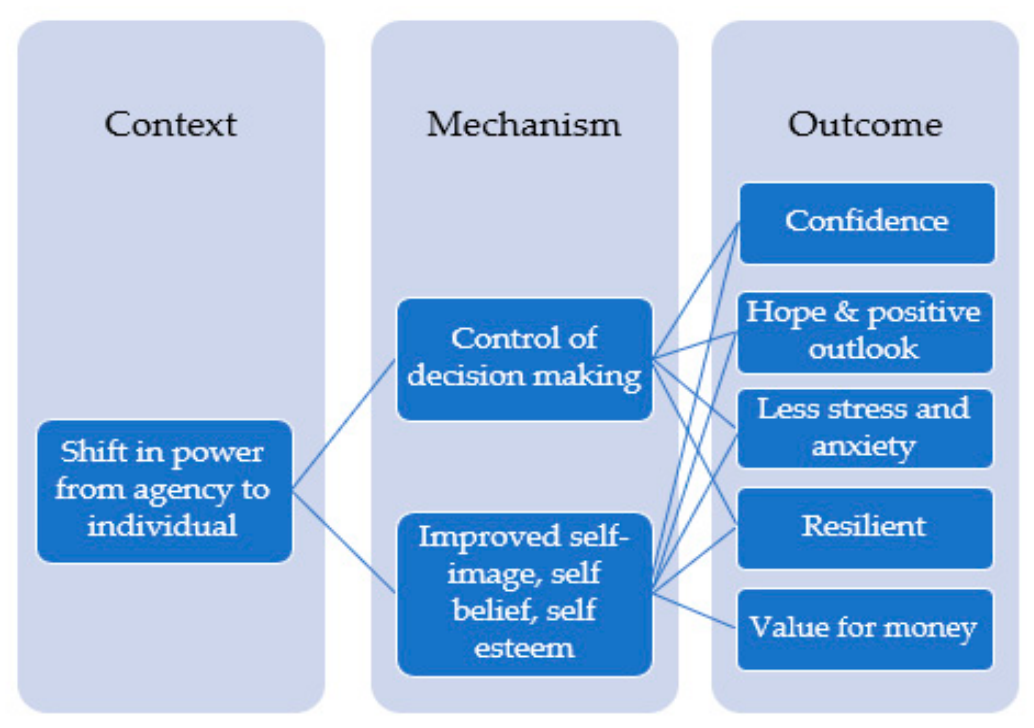

Figure 2. Meso CMO configuration. 
Another important theme- and also related to value for money-was the perception that people could avail of better opportunities in terms of social and recreational opportunities, getting outdoors, and being able to contribute to and integrate within society and the community through civic participation. An important factor here was the ability to purchase services from within mainstream, community-based settings, in turn increasing community integration and attendant experiences and opportunities. A key contextual factor here was the presence of a 'network of support' (Figure 3). In fact, when individuals were undergoing a 'suitability' assessment for individualised funding, staff often prioritised those who had access to a strong network of support. This typically comprised unpaid supports, such as family, friends, and colleagues, but our analysis clearly indicated that paid coordinators or support brokers (an intermediary between people with a disability and funding agencies) were also strongly associated with the person's network of support. The support also included sourcing information, recruiting staff, helping to broaden the person's network (often through community integration), and providing assistance with administrative and management tasks.

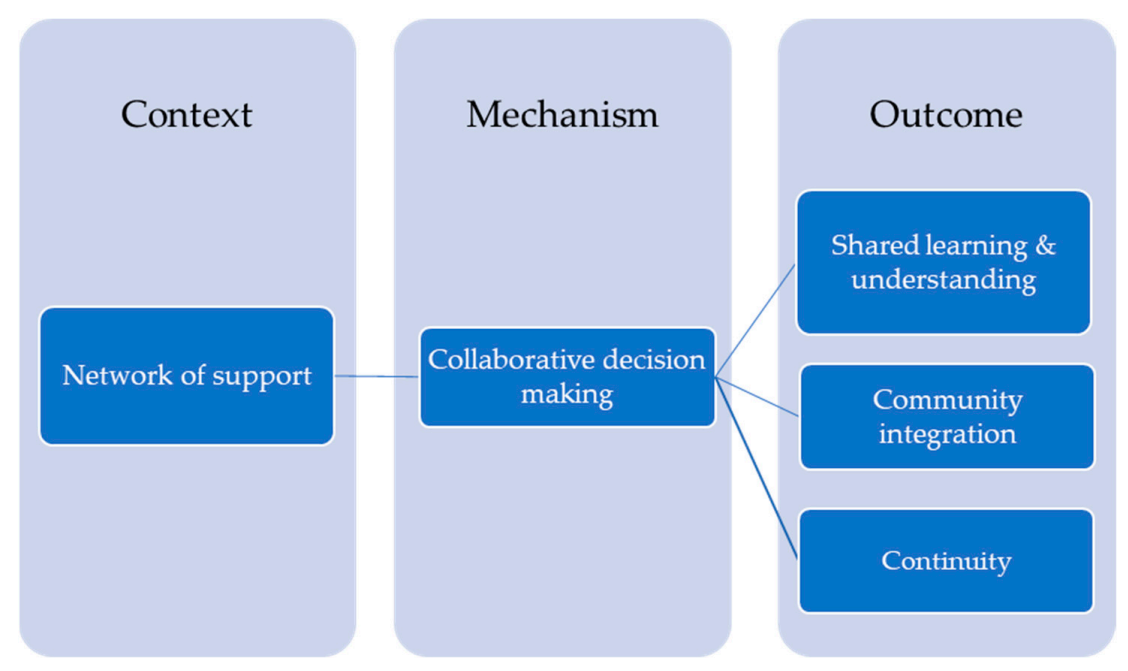

Figure 3. Meso CMO configuration.

The formal assessment of need was identified as both a key facilitative and inhibitive element of the individualised funding process and one which was often influenced by the presence of a network of support. Assessments, in some cases, were interpreted as a means to control or limit individuals, while in other circumstances, they were seen as genuinely needs-led. In the case of the latter, 'holistic or comprehensive' and 'outcome focused' approaches were highly valued-focusing on personal care, physical and mental health and wellbeing, social care, and quality of life. When people with a disability had strong advocates, assessment process tended to be more successful.

A strong network of support also led to collaborative relationships and decision making, which were also frequently identified as important mechanisms which led, in turn, to positive outcomes of shared learning and shared understanding - as well as continuity of care. These collaborations related to individuals and their informal/formal supporters as well as between agencies and government departments. People with a disability often spoke about personal assistants and their network of support having a better understanding as a result of individualised funding, while others recruited family members for the same reason (Figure 3).

The human resources that surround an individualised funding arrangement can promote or inhibit good practices, which, in turn, impacts outcomes. Frequently cited outcomes are related to people with a disability feeling less of a burden and having control over their lives, while also building valued and trusting relationships. A closely related mechanism was interpersonal relationships (Figure 4). This describes both personal 
attributes in terms of (a) the individual with a disability being proactive and open to new ideas and (b) providers thinking innovatively/creatively, as well as an overall approach to work that incorporates transparency, inclusivity, and positive risk taking.

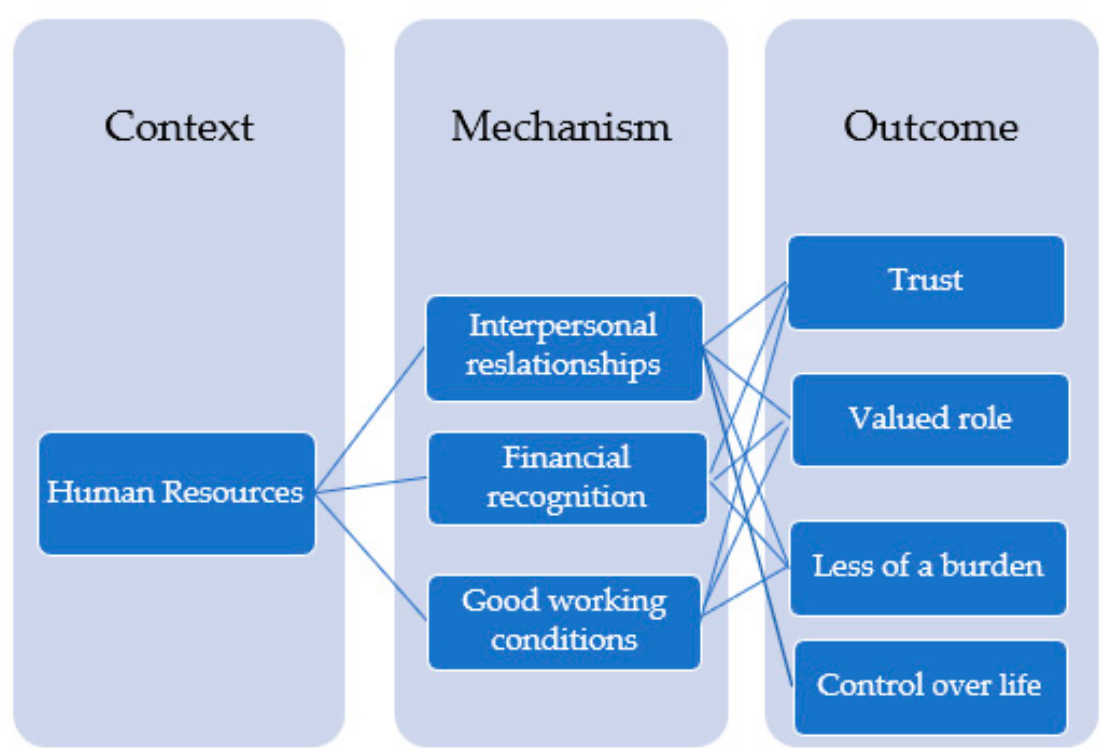

Figure 4. Micro CMO configuration.

The analysis revealed that financial recognition for voluntary work was one of the reasons why some people chose to take up individualised funding in the first instance. This was related to their ability to recruit family or friends, and sometimes meant that people with a disability no longer viewed themselves as a burden, since they were able to financially reward work that had previously been provided voluntarily. Furthermore, participants identified a number of mechanisms here as integral to success, which might collectively be considered as good working conditions, such as being a good employer. Treating staff well and offering an appropriate rate of payparticularly during unsocial working hours-was another element of support of particular value to people with a disability (Figure 4). To this end, the availability of training (for people with a disability/representatives), particularly around staff recruitment and management/administrative skills, was often cited as a facilitator of successful implementation. Furthermore, the analysis revealed that training was often suggested as a means of improving knowledge and understanding for staff.

\section{Discussion}

The evidence suggests that individualised funding is consistently being adopted and supported globally regardless of the theoretical, policy and practice intentions (or evidence base for effectiveness). It is well established that region-specific contextual factors, such as culture and policy, can influence access to and implementation of individualised funding. For example, in certain jurisdictions in Canada, the US, and the Netherlands, it is compulsory to use an independent support broker, whilst in the UK and the US, personal assistants are the preferred option for those receiving personal budgets. The eligibility criteria may also differ at initial implementation depending on the region. For example, in Canada, the focus is on younger people with learning disabilities, whereas the Swedes focus on adults with physical disabilities; furthermore, very few regions accommodate people with mental health problems. Objectives also differ; for example, Australia initially focused on tackling fragmented service provision, particularly in rural areas, while the US concentrated on solving staff shortages in long-term care facilities [24]. With these region-specific considerations in mind, it is clear how access to individualised funding (the first $\mathrm{CMO}$ configuration presented in this paper) varies considerably with different rules, 
regulations, and processes in place-with access ultimately dictated by policy decisions at the government level. These decisions impact on the mechanisms and outcomes that follow.

The available evidence highlights a number of benefits of individualised funding, but typically in the context of quantitative studies which focus on a limited range of outcomes (e.g., quality of life, client satisfaction) and which tend to be characterised by a number of common methodological limitations (e.g., lack of agreement on outcomes of interest, or how to measure them-leading to high levels of heterogeneity between studies). These studies, whilst useful and informative, do not explain how individualised funding achieves its intended goal of self-direction, empowerment, independence, and self-determination for people with a disability. This paper therefore attempts to identify-on the basis of the qualitative element of a recently conducted mixed methods systematic review-some key causal CMO configurations that might help to explain how these goals/outcomes may be achieved.

It is widely accepted, within the principles of realism, that the 'causes' of outcomes are not simple, linear or deterministic - instead, there is often a causal chain of CMOs [19]. Indeed, that is not to say that the mechanisms identified here are inherent to individualised funding, but rather they are a function of the participants and the contextual factors surrounding the individualised funding arrangement [19]. To this end, the CMO configurations presented in this paper are intended to identify some key causal factors that emerged from a large dataset representing a total 'sample' of approximately 9000 study participants. While most of the 69 qualitative studies in the review were very relevant $(87 \%)$, one potential limitation relates to the quality of included studies, with almost a third scoring 'low' or 'very low' in terms of methodological rigour (32\%) and risk of bias $(30 \%)$, whilst almost one quarter (23\%) were, likewise, considered to be limited in terms of coherence. To this end, a sensitivity analysis was conducted by removing studies with a very low CerQual score and comparing results to the analysis conducted with all studies included; this led to little or no change in the analysis [15].

Numerous challenges relating to individualised funding were identified within the review, both from the perspective of people with a disability and those providing them with support. Thus, it should be noted that while this paper focuses on contexts and mechanisms that support successful implementation, the absence of certain factors (such as a strong network of support), conversely, also inhibited implementation, depending on a person's own perspective, life experiences, and other contextual factors. For example, for one person, directly employing support workers might be perceived as empowering, whilst for another, it may be seen as stressful. This may be linked to socio-demographic factors; for example, an older person may have been using traditional forms of services for much longer than a young adult transitioning from mainstream school or another form of secondary education. Thus, past experiences, such as institutionalisation, may dramatically affect someone's ability to adapt to this new model of service provision. Equally, more people living in rural areas have been found to avail of day services when compared to urban dwellers, potentially due to a lack of alternatives within the community [20]. Similarly, while some people were empowered by moving away from agency-led support, others considered agencies to offer effective continuity of care, or to offer relief from the stress that was often associated with staff recruitment or general management of an individualised fund [15].

Despite these implementation challenges, individualised funding was widely accepted and positively viewed among people with a disability, as demonstrated by the wide range of benefits identified within the studies. These include quantitative metrics indicating improved quality of life and client satisfaction, as well as perceived benefits, including in particular, improvements in self-image and self-belief, a sense of empowerment, self-determination and confidence. With regard to the causal chain, having access to individualised funding is the most fundamental contextual factor that leads, through enhanced agency, to a sense of freedom and flexibility, which many people with a disability have not previously experienced within traditional models of services delivery. A close examination of the available literature within our review demonstrates that a significant 
contextual factor is the shift in power from agencies to the person with a disability or their representatives. For many people with a disability (and their families), this required approaching everyday tasks in a different way and taking control of the decisions around their day-to-day life, which led to a new perspective on their skills/abilities and self-image. With this renewed confidence, people reported a more positive outlook on life and opportunities, allowing them to subsequently pursue tangible changes that led to increased options and better-quality service provision.

As expected, the pathway to success differed depending on region-specific policies, such as overly complex and cumbersome bureaucracy. An important contextual factor that was consistently noted, however, was the presence (or possibility) of a strong network of support that utilised collaborative decision making between the funding recipient and funders/support agencies, often neutralising the aforementioned bureaucratic challenges. This was often achieved by building upon existing informal and formal relationships (to include extended family members, such as aunts, uncles, cousins, friends, and members of the wider community) through a process of co-produced goals and plans. Together, a shared vision emerged, mistakes were made, and lessons were learned collectively. Through this collaborative approach, real progress was achieved, often leading to community integration that, in itself, led to a wider organic network of support.

Careful consideration of the human resources surrounding an individualised funding arrangement prompted mechanisms that led to positive outcomes such as strengthening interpersonal relationships through increased recognition of people's crucial roles. This, in turn, fostered trust and enabled people with a disability and their network of support to commence a complex causal 'chain of events' that led to (varying degrees of) self-direction, empowerment, independence, and self-determination. As previous authors have noted, people with a disability do not necessarily have these kinds of skills and abilities [8,9], and therefore the literature clearly points to a need for upskilling and training.

It should be noted that the systematic review identified other contexts and mechanisms that led to, or indeed inhibited, positive outcomes for people availing of individualised funding. However, the $\mathrm{CMO}$ configurations presented in this paper were considered the most important, largely independent of disability-specific considerations, and should be helpful in terms of generating key generalisable lessons for those in the initial stages of implementing a programme of support based on the principles of individualised funding.

Supplementary Materials: The following are available online at https: / / www.mdpi.com/2673-727 2/1/1/4/s1, Qualitative Study Characteristics.

Author Contributions: Conceptualisation, P.F., S.M., S.T.; methodology, P.F., S.M., S.T.; validation, P.F., S.M., S.T.; formal analysis, P.F.; data curation, P.F.; writing—original draft preparation, P.F., S.M.; writing - review and editing, S.M., S.T.; visualisation, P.F.; supervision, S.M.; project administration, P.F. All authors have read and agreed to the published version of the manuscript.

Funding: This research was funded by Genio and conducted as part of the Health Research Board (HRB) Scholar Programme in Health Services Research (SPHeRE) [grant number PHD/2007/16].

Institutional Review Board Statement: The study was conducted according to the guidelines of the Declaration of Helsinki, and approved by the Ethics Committee of Maynooth University (SRESC2014-059, 5 November 2014).

Informed Consent Statement: Not applicable.

Data Availability Statement: The data presented in this study are openly available in the Wiley Online Library at https:/ / doi.org/10.4073/csr.2019.3 [see supporting information].

Acknowledgments: The authors would like to acknowledge the co-authors of the full systematic review and in particular Marian Hernon and Siobhan O'Doherty (RIP), who acted as second and third reviewers-in addition to their time and expertise, they generously offered encouragement and personal support through the very long systematic review process.

Conflicts of Interest: The authors declare no conflict of interest. 


\section{References}

1. United Nations. Convention on the Rights of Persons with Disabilities. 2006. Available online: http://www.un.org/disabilities/ convention/conventionfull.shtml (accessed on 25 May 2015).

2. WHO. Disability and health [Fact sheet N³52]. Media Centre 2013. Available online: http://www.who.int/mediacentre/ factsheets/fs352/en/ (accessed on 25 October 2014).

3. Perreault, S.; Vallerand, R.J. A test of self-determination theory with wheelchair basketball players with and without disability. Adapt. Phys. Act. Q. 2007, 24, 305-316. [CrossRef]

4. Saebu, M.; Sørensen, M.; Halvari, H. Motivation for physical activity in young adults with physical disabilities during a rehabilitation stay: A longitudinal test of self-determination theory. J. Appl. Soc. Psychol. 2013, 43, 612-625. [CrossRef]

5. Carr, S. Enabling risk and ensuring safety: Self-directed support and personal budgets. J. Adult Prot. 2010, 13, 122-136. [CrossRef]

6. Áiseanna, T. ÁT Philosophy. 2014. Available online: http://www.theatnetwork.com/about-us/at-philosophy/ (accessed on 16 October 2014).

7. NRCPDS. Cash \& Counseling. 2014. Available online: http://www.bc.edu/schools/gssw/nrcpds/cash_and_counseling.html/ (accessed on 30 October 2014).

8. Glendinning, C.; Kemp, P. Cash and Care: Policy Challenges in the Welfare State; Policy Press: Bristol, UK, 2006.

9. Fernandez, J.-L.; Kendall, J.; Davey, V.; Knapp, M. Direct payments in England: Factors linked to variations in local provision. J. Soc. Policy 2007, 36, 97-121. [CrossRef]

10. Fleming, P.; McGilloway, S.; Barry, S. Individualised funding for people with a disability in Ireland: The successes and challenges of implementing four pilot initiatives. In SPHeRE Network 2nd Annual Conference "Population Health and Health Services Research in Ireland: Current Trends and Future Directions"; SPHeRE Network, Royal College of Surgeons: Dublin, Ireland, 2016 ; p. 15.

11. Fixsen, D.; Naoom, S.; Blasé, K.; Friedman, R.; Wallace, F. Implementation Research: A Synthesis of the Literature; National Implementation Research Network, University of South Florida: Tampa, FL, USA, 2005.

12. Fleming, P.; Furlong, M.; McGilloway, S.; Keogh, F.; Hernon, M.; Stainton, T. Protocol: Personal budgeting interventions to improve health and social care outcomes for people with a disability: A systematic review. Campbell Syst. Rev. 2016. [CrossRef]

13. Brown, R.; Carlson, B.L.; Dale, S.; Foster, L.; Phillips, B.; Schore, J. Cash and Counseling: Improving the Lives of Medicaid Beneficiaries Who Need Personal Care or Home- and Community-Based Services Final Report; Mathimatica Policy Research Inc.: Princeton, NJ, USA, 2007.

14. Glendinning, C.; Challis, D.; Fernández, J.L.; Jacobs, S.; Jones, K.; Knapp, M.; Manthorpe, J.; Moran, N.; Netten, A.; Stevens, M.; et al. Evaluation of the Individual Budgets Pilot Programme: Final Report; Social Policy Research Unit, University of York: York, UK, 2008.

15. Fleming, P.; McGilloway, S.; Hernon, M.; Furlong, M.; O’Doherty, S.; Keogh, F.; Stainton, T. Individualized funding interventions to improve health and social care outcomes for people with a disability: A mixed-methods systematic review. Campbell Syst. Rev. 2019. [CrossRef]

16. Pawson, R.; Tilley, N. Realist Evaluation; Sage: London, UK, 1997.

17. Jagosh, J. Introduction to Realist Evaluation and Synthesis. Available online: https://youtu.be/QrneOfFXzx0 (accessed on 16 August 2017).

18. Astbury, B.; Leeuw, F.L. Unpacking black boxes: Mechanisms and theory building in evaluation. Am. J. Eval. 2010, 31, 363-381. [CrossRef]

19. Wong, G.; Westhorp, G.; Pawson, R.; Greenhalgh, T. Realist Synthesis RAMESES Training Materials. Available online: https: / / www.ramesesproject.org/media/Realist_reviews_training_materials.pdf (accessed on 12 January 2021).

20. Fleming, P.; McGilloway, S.; Barry, S. The successes and challenges of implementing individualised funding and supports for disabled people: An Irish perspective. Disabil. Soc. 2016, 31, 1369-1384. [CrossRef]

21. Laragy, C.; Ottmann, G. Towards a framework for implementing individual funding based on an Australian case study. J. Policy Pract. Intellect. Disabil. 2011, 8, 18-27. [CrossRef]

22. MAXQDA. Qualitative Data Analysis Software for Mac and Windows [Computer Software]; VERBI GmbH: Berlin, Germany, 2014.

23. Malterud, K.; Siersma, V.D.; Guassora, A.D. Sample size in qualitative interview studies: Guided by information power. Qual. Health Res. 2016, 26, 1753-1760. [CrossRef] [PubMed]

24. Carr, S.; Robbins, D. SCIE Research Briefing 20: The Implementation of Individual Budget Schemes in Adult Social Care; Social Care Institute for Excellence: London, UK, 2009. 